\title{
Cancer-COVID-19 Mortality Prediction:- An Algorithm by Bayesian Autoregressive Model
}

\author{
Atanu Bhattacharjee \\ Section of Biostatistics, Centre for Cancer Epidemiology, \\ Tata Memorial Centre, Mumbai,India \\ Hombi Bhaba National Institute,Mumbai,India
}

April 23, 2020

\section{Abstract}

This pandemic of COVID-19 is tedious to control. The only lockdown is the way to stop the spread of this infection. Conventional health care is facing a real challenge to operate. Primarily the challenge is to provide health care support for COVID-19 patients with limited resources and continue the health care services like earlier.Perhaps, this challenge is the same but magnitude is different from different geographical locations around the globe. In this article, we presented a Bayesian algorithm with the Code to predict cancer death due to COVID-19. This code is possible to run at different time points and different geographical locations around the world. This code will help us to get the best strategy and shift the treatment option for cancer treatment. The model would provide physicians with an objective tool for counseling and decision making at different hotspots and small areas to implement.

Keywords:-COVID-19; Cancer; Decision; Bayesian, autoregressive model;

\section{Introduction}

In this current COVID-19 pandemic, we are waking up to the limitations of their analog health care system. We need an immediate digital solution, 
pursued on several fronts, to address this crisis. It is crucial to implement predicting maps during this outbreak and attain all possible ways to explore an estimation of transmission rate who infected whom, where, when and what the rate? But it is difficult to understand the transmission because it is not known always.We can find out when a particular host individual contacted the infection, and thereafter transmitted to others. It is required to uncover the transmission process case to cases to create dynamic models.

Lockdown is the only solution to stop the COVID-19 pandemic. We do not have sufficient data with cancer and COVID-19. Are cancer patients prone to get infected? Do they have more complications? The consequence of infection is death. What is the risk of death due to COVID-19 among cancer patients? Broadly, any active treatment is a management of cancer by prolonging death occurrence. Similarly, lockdown is to halt the infection and deaths. Now the concern is about to halt the treatment for cancer or continue it? We preferred to perform the Bayesian autoregressive modeling. A total of 28 days of India's COVID-19 confirmed and mortality data is considered. The autoregressive model is used to predict the next 50 days' projections on mortality and confirmed cases for the general population and cancer patients. The simulated data has generated the predict the cancer COVID-19 death for illustration.

The univariate Bayesian approach is an already established method. It is possible to obtain parameter estimation by iterative generalized least square $[6,7]$. The most common approach is the generalize the conditional autoregressive (CAR) distribution [1]. The multivariate approach is already explored [2-5].Ths autoregressive approach is valid to predict the COVID-19 disease. We apply the autoregressive model in the COVID-19 India model.

The objective of this work is to predict the appearance of COVID-19 related death among cancer patients. The data visualization technique is utilized to represent the gravity of the problem. The linear and non-linear autoregressive modeling is performed. The National level data available of COVID-19 confirmed and death counts are considered to predict the COVID-19 case count and death for the next 50 days. Similarly, the same model is utilized with prior information on national data to predict cancer patients' death and confirmed cases for the next 50 days. We provide the code that will help us to get the best strategy and shift the treatment option for cancer treatment at a time to time basis. The model would provide physicians with an objective tool for counseling and decision making at different hotspots and small areas 
to implement. The model is easy to run in OpenBUG software to generate the prediction. The OpenBUG software is opensource. It is possible to generate prediction with convergence with updated real time data.

\section{Statistical Model}

\section{Linear Autoregressive Regression}

A linear model is defined as

$$
Y(t)=\beta_{0}+\beta_{1} X_{1}(t)+\beta_{2} X_{2}(t)=\ldots+\beta_{m} X_{m}(t)+Z(t)
$$

It is defined as $Y(t)$ as the observation of the dependent variable at time $t$. Now $X_{i}(t)$ is presented as the i-th independent variable at time $t$, and finally $Z(t)$ is the error term at time $t$. The errors $Z(t) ; t=1,2 \ldots, n$ term is assumed with mean zero ,have a constant variance, and autocorrelated. Now objective is to compute the Bayesain inference for the $m$ with regression coefficient $\beta_{i}$. The unknown parameters of the error process. It is assumed that $Z(t)$ to follow $\mathrm{AR}(1)$ with autocorrelation $\theta$.

\section{Non-linear Autoregressive Model}

However, the number of cases count is unknown in the future. It may progress by non-linear trends. The non-linear autoregressive modeling is defined as

$$
Y(t)=\exp \left(\beta_{0}+\beta_{1} t\right)+Z(t)
$$

Similarly, the intercept is presented as $\beta_{0}$ and the regression parameter is $\beta_{1}$ are not known. The corresponding error for this model is estimated at $Z(t)$. The autocorrelation $\theta$ with $\mathrm{AR}(1)$ process is assumed. The OpenBUG code is presented below. Suppose the observations is assumed as $t$. The Bayesian computation is performed with 28 observations i.e. 4 weeks count data. It helped to estimates of $\beta_{0}, \beta_{1}$ and $\theta$, and $\sigma^{2}$ respectively. A total of 30,000 iterations for simulation and 5,000 burn-in-was performed. The observation with outcome $Y$ is assumed with a normal distribution. The variance-covariance matrix of $\mathrm{AR}(1)$ with correlation $\theta$ is defined. The posterior estimate is computed through simulation. 


\section{Result}

Indian confirmed, recovered and death count data are presented (Figure1) for the last 28 days. It shows the real and overwhelming success of lockdown to retains the case count with limited numbers. Perhaps, death is contributed to a marginal proportion. The available data on https://www.mohfw.gov.in/ website is presented. It presents a comparison between the proportion of death and recovery among confirmed cases (Figure 2 (A)). Similarly, a 10\% higher rate of death among cancer patients with similar recovery rates is presented (Figure 2(B)). Further, we applied the Bayesian auto-regressive model. The autoregressive model is separated into linear and non-linear models. The linear model is defined in equation(1). Similarly, the non-linear model is presented in equation (2).

The posterior estimates of the regression coefficients are obtained by the linear autoregressive model. The model is performed for confirmed, recovered and died cases separately. The result of posterior estimates is presented in Table 1. A total of four parameters are estimated. The $\beta_{0}$ and $\beta_{1}$ represents the intercept and regression coefficients. The variance of the model is presented by $v$. the amount of autocorrelation is estimated by $\theta$. A total of 30,000 iterations are performed to obtain convergence and estimates. Now the linear autoregressive model is defined as Model 1 (for confirmed cases), Model 2( for died case) and Model 3( recovered cases). The linear model simulation is presented in Figure 4. It shows to obtain the converges for the parameters. The posterior estimates of the non-linear model are presented in Table 2. The posterior regression estimates of all the parameters can be used for prediction of the case and death count for the next 50 days (figure 3).The OpenBUG code is presented below can be used to obtain the prediction. It shows that the death count among cancer patients is relatively high. The treatment provision with lockdown may challenge cancer death. Cancer patients may die due to COVID-19 infection and simultaneously without treatment due to cancer.

\section{Conclusion}

This document aimed to provide a practical scenario in presence of COVID19 infection spread among cancer patients. The possible impact of patients in Head and neck cancer. It is required to judge very crucially cases to case 
basis about providing the treatment and reduce mortality due to cancer. Because the attempt to reduce mortality due to cancer can incline death due to COIVD-19 and spread of infection. The spread of infection will also put other non-cancer individuals into a risk. We hope to help head and neck cancer patients to survive this crucial period.

\section{Acknowledgement}

None.

\section{Conflict of interest}

No

Table 1: Linear Autoregressive Posterior Estimates of Confirmed, deceased and recovered cases on prediction.

\begin{tabular}{|c|c|c|c|}
\hline Parameter & Mean & $\mathrm{SD}$ & $95 \%(\mathrm{HPD})$ \\
\hline \multicolumn{4}{|c|}{ Indian states Confirmed cases } \\
\hline$\beta_{0}$ & 4.38 & 0.44 & $(3.56,5.11)$ \\
\hline$\beta_{1}$ & 0.11 & 0.01 & $(0.08,0.14)$ \\
\hline$\theta$ & 0.92 & 0.04 & $(0.82,0.97)$ \\
\hline $\mathrm{V}$ & 3527 & 1164 & $(1873,6366)$ \\
\hline \multicolumn{4}{|c|}{ Indian states Deceased cases } \\
\hline$\beta_{0}$ & 0.38 & 0.52 & $(-0.72,1.51)$ \\
\hline$\beta_{1}$ & 0.13 & 0.02 & $(0.09,0.17)$ \\
\hline$\theta$ & 0.71 & 0.17 & $(0.34,0.95)$ \\
\hline $\mathrm{v}$ & 46.29 & 27.33 & $(10.29,110.9$ \\
\hline \multicolumn{4}{|c|}{ Indian states Recovered cases } \\
\hline$\beta_{0}$ & -0.76 & 0.98 & $(-0.71,1.41)$ \\
\hline$\beta_{1}$ & 0.22 & 0.03 & $(0.14,0.30)$ \\
\hline$\theta$ & 0.74 & 0.17 & $(0.36, .96)$ \\
\hline $\mathrm{V}$ & 307.3 & 191.50 & $(77.96,767)$ \\
\hline
\end{tabular}


Table 2: Non-linear Autoregressive Posterior Estimates of Confirmed, deceased and recovered cases on prediction.

\begin{tabular}{|c|c|c|c|}
\hline Parameter & Mean & $\mathrm{SD}$ & $95 \%(\mathrm{HPD})$ \\
\hline \multicolumn{4}{|c|}{ Indian states Confirmed cases } \\
\hline$\beta_{0}$ & 4.38 & 0.44 & $(3.56,5.11)$ \\
\hline$\beta_{1}$ & 0.11 & 0.01 & $(0.08,0.14)$ \\
\hline$\theta$ & 0.92 & 0.04 & $(0.82,0.97)$ \\
\hline $\mathrm{v}$ & 3527 & 1164 & $(1873,6366)$ \\
\hline \multicolumn{4}{|c|}{ Indian states Deceased cases } \\
\hline$\beta_{0}$ & 0.38 & 0.52 & $(-0.72,1.51)$ \\
\hline$\beta_{1}$ & 0.13 & 0.02 & $(0.09,0.17)$ \\
\hline$\theta$ & 0.71 & 0.17 & $(0.34,0.95)$ \\
\hline $\mathrm{V}$ & 46.29 & 27.33 & $(10.29,110.9)$ \\
\hline \multicolumn{4}{|c|}{ Indian states Recovered cases } \\
\hline$\beta_{0}$ & -0.76 & 0.98 & $(-2.83,1.41)$ \\
\hline$\beta_{1}$ & 0.22 & 0.03 & $(0.14,0.30)$ \\
\hline$\theta$ & 0.74 & 0.1711 & $(0.36,0.96)$ \\
\hline$v$ & 307.3 & 191.5 & $(77.96,767)$ \\
\hline
\end{tabular}

\section{OpenBUGS Model}

model

\{

beta $0 \sim \operatorname{dnorm}(0, .001)$

beta1 $\sim \operatorname{dnorm}(0, .001)$

theta $\sim \operatorname{dbeta}(6,4)$

$\mathrm{v} \sim \operatorname{dgamma}(.001, .001)$

for $(\mathrm{t}$ in $1: 28)\{\mathrm{mu}[\mathrm{t}]<-\exp ($ beta $0+$ beta $1 * \mathrm{t})$

$\mathrm{Y}[1,1: 28] \sim \operatorname{dmnorm}(\mathrm{mu}[], \operatorname{tau}[]$,

$\operatorname{tau}[1: 28,1: 28]<$-inverse(Sigma[,])

for $(\mathrm{i}$ in $1: 28)\{$ Sigma $[\mathrm{i}, \mathrm{i}]<-\mathrm{v} /(1$-theta*theta $)$

for(i in 1:28)\{for(j in i+1:28)Sigma $[\mathrm{i}, \mathrm{j}]<-\mathrm{v}^{*}$ pow $($ theta, $\mathrm{j}) * 1 /$

(1-theta*theta) $\}\}$

for( $\mathrm{i}$ in 2:28) for( $\mathrm{j}$ in 1: i-1)Sigma $[\mathrm{i}, \mathrm{j}]<-\mathrm{v}^{*} \operatorname{pow}($ theta, $\mathrm{i}-1) * 1 /$

(1-theta*theta) $\}$ \}

Indian states Confirmed cases

list(

6

$\mathrm{Y}=$ structure $($ Data $=\mathrm{c}(158,56,28,28,72,54,116,156,134,204,128,180,130$, $328,286,220,380,612,848,972,1120,1158,1210,978,1146,1130,1626,1742)$,

$. \operatorname{Dim}=c(1,28)))$

list $($ beta $0=1$, beta $1=.05$, theta $=.6, \mathrm{v}=4)$ 


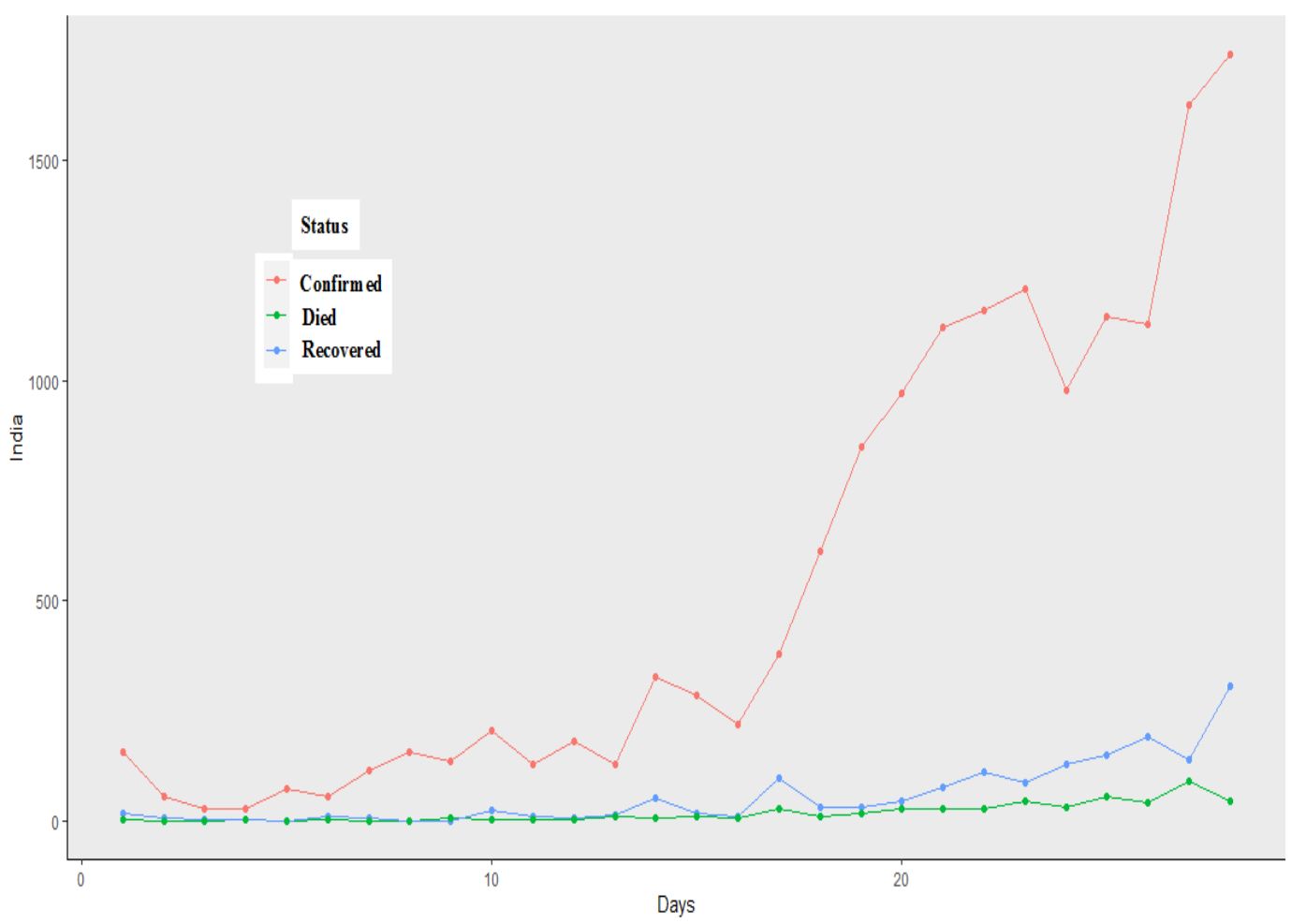

Figure 1: The comparison between the official COVID-19 pandemic data of confirmed, died and recovered. cases in India.

\section{Prediction of Confirmed cases from posterior regression estimates}

set.seed $(1)$

$\mathrm{w}<-\operatorname{rnorm}(100, \mathrm{sd}=59.38)$

$\mathrm{z}<-\operatorname{rep}(0,100)$

for $(\mathrm{t}$ in $2: 100) \mathrm{z}[\mathrm{t}]<-0.92{ }^{*} \mathrm{z}[\mathrm{t}-1]+\mathrm{w}[\mathrm{t}]$

Time $<-1: 100$

$\mathrm{f}<-$ function $(\mathrm{x}) \exp \left(4.38+0.11^{*} \mathrm{x}\right)$

$\mathrm{x}<-\mathrm{f}($ Time $)+\mathrm{z}$

plot(x,ylab="India Confirmed cases",xlab="Days") 

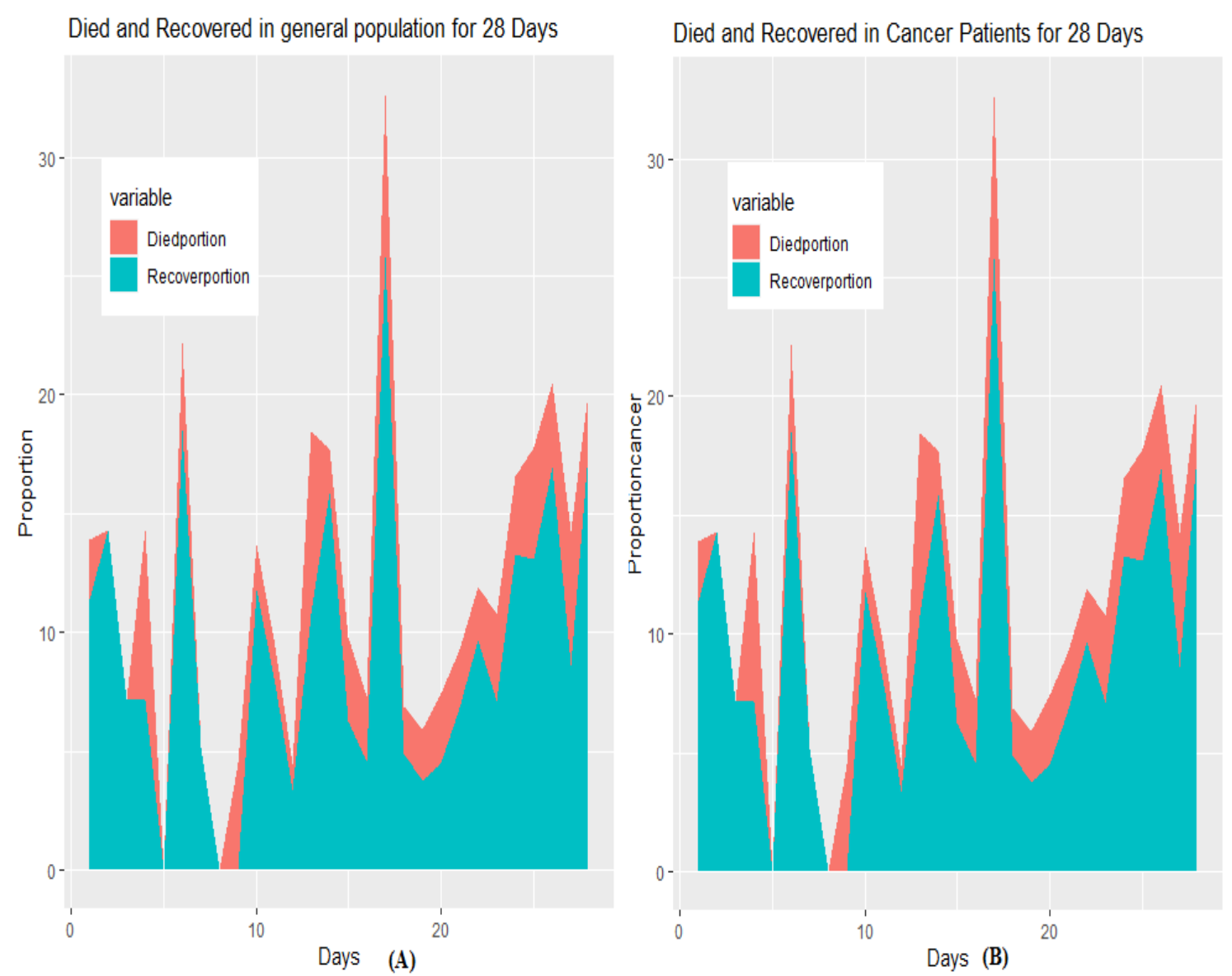

Figure 2: The comparison between died and recovered proportion in general population(A) and cancer patients(B).

\section{References}

[1] Julian Besag. Spatial interaction and the statistical analysis of lattice systems. Journal of the Royal Statistical Society: Series B (Methodological), 36(2):192-225, 1974.

[2] Alan E Gelfand and Penelope Vounatsou. Proper multivariate conditional autoregressive models for spatial data analysis. Biostatistics, 4(1):11-15, 2003. 

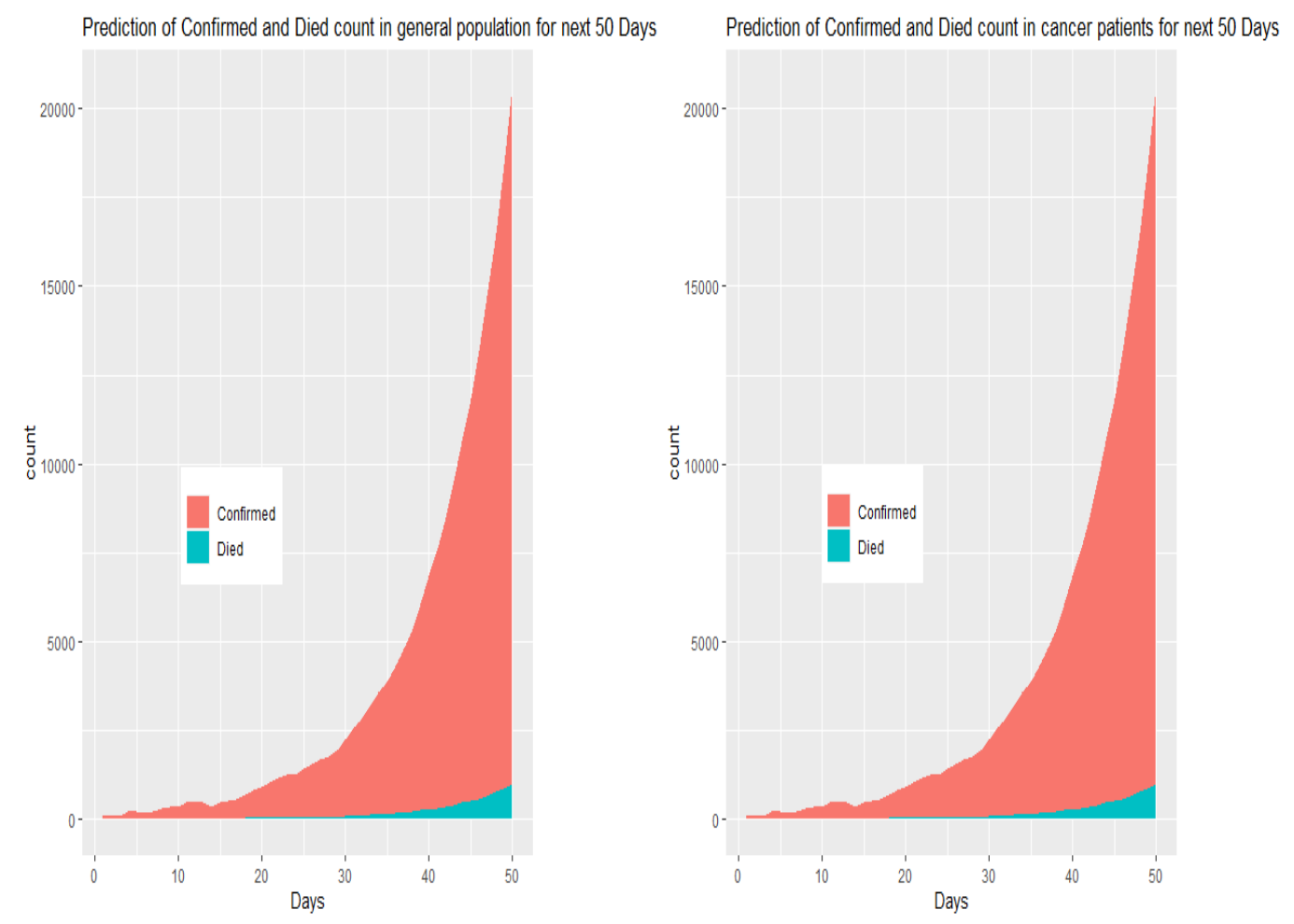

Figure 3: The prediction of case count and death count for next 50 days in general population and cancer patients.

[3] W Keith Hastings. Monte carlo sampling methods using markov chains and their applications. 1970.

[4] Xiaoping Jin, Sudipto Banerjee, and Bradley P Carlin. Order-free coregionalized areal data models with application to multiple-disease mapping. Journal of the Royal Statistical Society: Series B (Statistical Methodology), 69(5):817-838, 2007.

[5] Hoon Kim, Dongchu Sun, and Robert K Tsutakawa. A bivariate bayes method for improving the estimates of mortality rates with a twofold conditional autoregressive model. Journal of the American Statistical association, 96(456):1506-1521, 2001.

[6] Ian H Langford, Alistair H Leyland, Jon Rasbash, and Harvey Goldstein. Multilevel modelling of the geographical distributions of diseases. Journal 

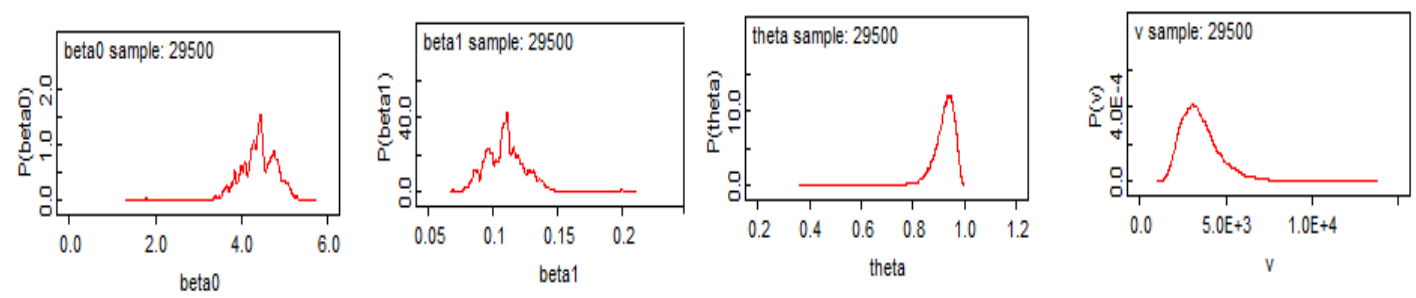

\section{Model1}
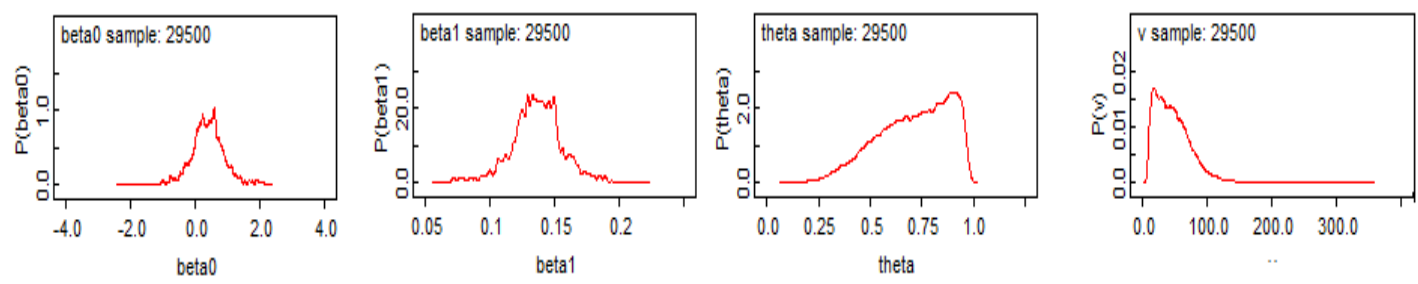

\section{Model2}
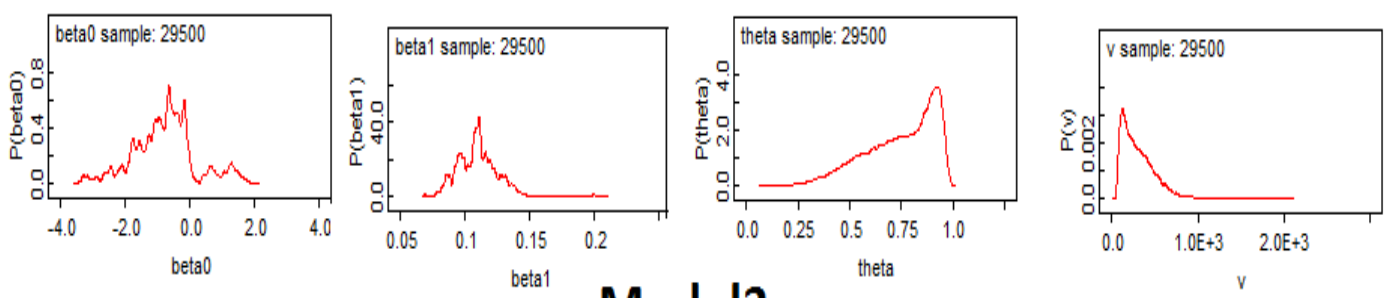

Figure 4: Iteration convergence of different parameters.

of the Royal Statistical Society: Series C (Applied Statistics), 48(2):253268, 1999.

[7] Alastair H Leyland, Ian H Langford, Jon Rasbash, and Harvey Goldstein. Multivariate spatial models for event data. Statistics in Medicine, 19(1718):2469-2478, 2000. 